TAIWANESE JOURNAL OF MATHEMATICS

Vol. 19, No. 1, pp. 193-209, February 2015

DOI: $10.11650 /$ tjm.19.2015.3856

This paper is available online at http://journal.taiwanmathsoc.org.tw

\title{
ON THE CONVERGENCE OF A MODIFIED CHEBYSHEV-LIKE'S METHOD FOR SOLVING NONLINEAR EQUATIONS
}

\author{
Lin Zheng*, Ke Zhang and Liang Chen
}

\begin{abstract}
In this paper, we introduce a modified Chebyshev-like's method with order four and study the semilocal convergence of the method by using majorizing functions for solving nonlinear equations in Banach spaces. We prove an existence-uniqueness theorem and give a priori error bounds which demonstrates the R-order of the method. Moveover, the local convergence of this method is also analyzed. Finally, numerical application on nonlinear integral equations is given to show our approach.
\end{abstract}

\section{INTRODUCTION}

Many scientific and engineering problems can be brought in the form of a nonlinear equation

$$
F(x)=0,
$$

where $F$ is a third order Fréchet-differentiable operator defined on a convex subset $\Omega$ of a Banach space $X$ with values in a Banach space $Y$.

There are kinds of methods to find a solution of Equation (1). Generally, iterative methods are often used to solve this problem ([1]). The most well-known iterative method is Newton's method

$$
x_{n+1}=x_{n}-F^{\prime}\left(x_{n}\right)^{-1} F\left(x_{n}\right),
$$

Received September 30, 2013, accepted May 8, 2014.

Communicated by Wen-Wei Lin.

2010 Mathematics Subject Classification: 65J15, 65H10, 65G99, 47J25, 49M15.

Key words and phrases: Nonlinear equations, Semilocal convergence, Chebyshev-like's method, Local convergence, Banach spaces.

The work are supported by National Natural Science Foundation of China (11371243, 11301001, 61300048), Innovation Program of Shanghai Municipal Education Commission (13ZZ068), Anhui Provincial Natural Science Foundation (1308085QF117), Natural Science Foundation of Universities of Anhui Province (KJ2011A248, KJ2012Z347), The Open Fund of Shanghai Key Laboratory of Trustworthy Computing (07DZ22304201004), Excellent Youth Scholars Foundation and the Natural Science Foundation of Anhui Province(2013SQRL030ZD) and The Young Foundation of Huaibei Normal University (2012XQ42).

*Corresponding author. 
which has quadratic convergence. Recently a lot of research has been carried out to provide improvements. Third-order iterative methods such as Halley's method, Chebyshev's method, super-Halley's method, etc [2-10] are used to solve Equation (1). To improve the convergence order, fourth-order iterative methods are also discussed in [1115]. The convergence of these iterative methods in Banach spaces is established by using recurrence relations. An alternative approach is developed to establish this convergence by using majorizing functions. The approach is also a very popular technique to establish the convergence of iterative methods. For example, it has been successfully applied to the convergence analysis of Newton's method and some high-order methods [16-27].

Ezquerro, etc studied a Chebyshev-like's method in [28], which is given by the following algorihtm

$$
x_{n+1}=x_{n}-\left[I+\frac{1}{2} L_{F}\left(x_{n}\right)+\frac{1}{2} L_{F}^{2}\left(x_{n}\right)\right] \Gamma_{n} F\left(x_{n}\right),
$$

where $\Gamma_{n}=\left[F^{\prime}\left(x_{n}\right)\right]^{-1}$ and $L_{F}\left(x_{n}\right)=\Gamma_{n} F^{\prime \prime}\left(x_{n}\right) \Gamma_{n} F\left(x_{n}\right)$. Method (3) has R-order of convergence at least three, but if $F$ is a quadratic operator, its R-order of convergence is at least four ([29]).

Kou, etc [30] presented a variant of the super-Halley method which improves the order of the super-Halley method from three to four by using the values of the second derivative at $\left(x_{n}-\frac{1}{3} f\left(x_{n}\right) / f^{\prime}\left(x_{n}\right)\right)$ instead of $x_{n}$. Wang, etc [12] established the semilocal convergence of the fourth-order super-Halley method in Banach spaces by using recurrence relations. This method in Banach spaces can be given by

$$
x_{n+1}=x_{n}-\left[I+\frac{1}{2} K_{F}\left(x_{n}\right)\left[I-K_{F}\left(x_{n}\right)\right]^{-1}\right] \Gamma_{n} F\left(x_{n}\right),
$$

where $\Gamma_{n}=\left[F^{\prime}\left(x_{n}\right)\right]^{-1}, K_{F}\left(x_{n}\right)=\Gamma_{n} F^{\prime \prime}\left(u_{n}\right) \Gamma_{n} F\left(x_{n}\right)$ and $u_{n}=x_{n}-\frac{1}{3} \Gamma_{n} F\left(x_{n}\right)$.

In [27], we establish the Newton-Kantorovich-type convergence theorem for the method (4) by using majorizing functions. Motivated by the super-Halley method (4), we introduce a modified Chebyshev-like's method with fourth-order in this paper and consider the convergence of the method which can be written as

$$
x_{n+1}=x_{n}-\left[I+\frac{1}{2} K_{F}\left(x_{n}\right)+\frac{1}{2} K_{F}^{2}\left(x_{n}\right)\right] \Gamma_{n} F\left(x_{n}\right),
$$

where $\Gamma_{n}=\left[F^{\prime}\left(x_{n}\right)\right]^{-1}, K_{F}\left(x_{n}\right)=\Gamma_{n} F^{\prime \prime}\left(u_{n}\right) \Gamma_{n} F\left(x_{n}\right), y_{n}=x_{n}-\Gamma_{n} F\left(x_{n}\right)$ and $u_{n}=x_{n}+\frac{1}{3}\left(y_{n}-x_{n}\right)$.

Compared with the Chebyshev-like's method in [28], this method requires nearly the same costs, but the convergence order is increased to at least four. Therefore the modified Chebyshev-like's method can be more efficient and of practical interest.

The basic advantage of this method is that, as the matrix that appears at each iteration is the same, the number of associated linear systems that we need to solve 
is smaller. This happens because, at each iteration, only one LR decomposition is computed. In most cases, the computational cost of solving a linear system is more expensive than that of the evaluations of the operator.

The modified Chebyshev-like's iterative method (5) requires in the finite dimensional case the computations which appear in Table 1. We also compare it with the super-Halley method (4) and the Chebyshev-like's method (3). We observe that the modified Chebyshev-like's method (5) can be superior.

Table 1: Computational cost and R-order of convergence

\begin{tabular}{cccccc}
\hline Method & R-order & Ev. of $F$ & Ev. of $F^{\prime}$ & Ev. of $F^{\prime \prime}$ & LR-dec. \\
\hline Method (3) & 3 & 1 & 1 & 1 & 1 \\
Method (4) & 4 & 1 & 1 & 1 & 2 \\
Method (5) & 4 & 1 & 1 & 1 & 1 \\
\hline
\end{tabular}

We shall use majorizing functions to prove the semilocal convergence for modified Chebyshev-like's method with order four. Based on it, an existence-uniqueness theorem is given to establish the R-order of the method to be four and a priori error bounds. Furthermore, we also analyse the local convergence of this method. In the end, numerical example is presented to demonstrate our approach.

\section{Semilocal Convergence}

Definition $^{[1]}$ Let $\Pi$ be an iterative process for the nonlinear operator $F$ with limit point $x^{*}$. The $\mathrm{R}$-order of $\Pi$ is defined by the quantity

$$
O_{R}\left(\Pi, x^{*}\right)=\left\{\begin{array}{c}
\infty, \text { if } R_{q}\left(\Pi, x^{*}\right)=0, \forall q \in[1, \infty), \\
\inf \left\{q \in[1, \infty) \mid R_{q}\left(\Pi, x^{*}\right)=1\right\}, \text { otherwise, }
\end{array}\right.
$$

where

$$
R_{q}\left(\Pi, x^{*}\right)=\left\{\begin{array}{l}
\lim \sup _{n \rightarrow \infty}\left\|x_{n}-x^{*}\right\|^{1 / n}, \text { if } q=1, \\
\limsup _{n \rightarrow \infty}\left\|x_{n}-x^{*}\right\|^{1 / n}, \text { if } q>1 .
\end{array}\right.
$$

If $0<R_{q}\left(\Pi, x^{*}\right)<1$ holds for some $q \in[1, \infty)$, then the R-order of convergence of $\Pi$ is $q$.

We firstly give an approximation of the operator $F$ in the following lemma, which will be used in the next derivation.

Lemma 1. Assume that the nonlinear operator $F: \Omega \subset X \rightarrow Y$ is continuously third-order Fréchet differentiable where $\Omega$ is an open set and $X$ and $Y$ are Banach 
spaces, and the sequence $\left\{x_{n}\right\}$ generated by (5) is well defined. Then we have

(6)

$$
\begin{aligned}
F\left(x_{n+1}\right) & =-\frac{1}{2}\left[F^{\prime \prime}\left(u_{n}\right)-F^{\prime \prime}\left(x_{n}\right)\right]\left(y_{n}-x_{n}\right) K_{F}\left(x_{n}\right)\left(y_{n}-x_{n}\right) \\
& +\frac{1}{2} F^{\prime \prime}\left(x_{n}\right)\left(y_{n}-x_{n}\right) K_{F}^{2}\left(x_{n}\right)\left(y_{n}-x_{n}\right) \\
& +\int_{0}^{1} F^{\prime \prime}\left(y_{n}+t\left(x_{n+1}-y_{n}\right)\right)(1-t) d t\left(x_{n+1}-y_{n}\right)^{2} \\
& -\frac{1}{6} \int_{0}^{1}\left[F^{\prime \prime \prime}\left(x_{n}+\frac{1}{3} t\left(y_{n}-x_{n}\right)\right)-F^{\prime \prime \prime}\left(x_{n}\right)\right] d t\left(y_{n}-x_{n}\right)^{3} \\
& +\frac{1}{2} \int_{0}^{1}\left[F^{\prime \prime \prime}\left(x_{n}+t\left(y_{n}-x_{n}\right)\right)-F^{\prime \prime \prime}\left(x_{n}\right)\right](1-t)^{2} d t\left(y_{n}-x_{n}\right)^{3} \\
& +\int_{0}^{1} F^{\prime \prime \prime}\left(x_{n}+t\left(y_{n}-x_{n}\right)\right)(1-t) d t\left(y_{n}-x_{n}\right)^{2}\left(x_{n+1}-y_{n}\right),
\end{aligned}
$$

where $y_{n}=x_{n}-F^{\prime}\left(x_{n}\right)^{-1} F\left(x_{n}\right)$ and $u_{n}=x_{n}+\frac{1}{3}\left(y_{n}-x_{n}\right)$.

Proof. By Taylor expansion, we have

$$
F\left(x_{n+1}\right)=F\left(y_{n}\right)+F^{\prime}\left(y_{n}\right)\left(x_{n+1}-y_{n}\right)
$$

$$
\begin{aligned}
F\left(y_{n}\right)= & \frac{1}{2} F^{\prime \prime}\left(x_{n}\right)\left(y_{n}-x_{n}\right)^{2}+\frac{1}{6} F^{\prime \prime \prime}\left(x_{n}\right)\left(y_{n}-x_{n}\right)^{3} \\
& +\frac{1}{2} \int_{0}^{1}\left[F^{\prime \prime \prime}\left(x_{n}+t\left(y_{n}-x_{n}\right)\right)-F^{\prime \prime \prime}\left(x_{n}\right)\right](1-t)^{2} d t\left(y_{n}-x_{n}\right)^{3},
\end{aligned}
$$

and

$$
F^{\prime}\left(y_{n}\right)=F^{\prime}\left(x_{n}\right)+F^{\prime \prime}\left(x_{n}\right)\left(y_{n}-x_{n}\right)
$$

$$
+\int_{0}^{1} F^{\prime \prime \prime}\left(x_{n}+t\left(y_{n}-x_{n}\right)\right)(1-t) d t\left(y_{n}-x_{n}\right)^{2} \text {. }
$$

We also noticed that

$$
\begin{aligned}
F^{\prime \prime}\left(x_{n}\right)= & F^{\prime \prime}\left(u_{n}\right)-\frac{1}{3} F^{\prime \prime \prime}\left(x_{n}\right)\left(y_{n}-x_{n}\right) \\
& -\frac{1}{3} \int_{0}^{1}\left[F^{\prime \prime \prime}\left(x_{n}+\frac{1}{3} t\left(y_{n}-x_{n}\right)\right)-F^{\prime \prime \prime}\left(x_{n}\right)\right] d t\left(y_{n}-x_{n}\right) .
\end{aligned}
$$

Substituting (8)-(10) into (7), we can obtain (6). 
Let $x_{0} \in \Omega$ and the nonlinear operator $F: \Omega \subset X \rightarrow Y$ be continuously third order Fréchet differentiable where $\Omega$ is an open set and $X$ and $Y$ are Banach spaces. Assume that

(C1) $\left\|\Gamma_{0} F\left(x_{0}\right)\right\| \leq \eta$,

(C2) $\left\|\Gamma_{0}\right\| \leq \beta$,

(C3) $\left\|F^{\prime \prime}(x)\right\| \leq M, x \in \Omega$,

(C4) $\left\|F^{\prime \prime \prime}(x)\right\| \leq N, x \in \Omega$,

(C5) there exists a positive real number $L$ such that

$$
\left\|F^{\prime \prime \prime}(x)-F^{\prime \prime \prime}(y)\right\| \leq L\|x-y\|, \forall x, y \in \Omega .
$$

Denote

$$
g(t)=\frac{1}{2} K t^{2}-\frac{t}{\beta}+\frac{\eta}{\beta},
$$

where $K$ are the positive real numbers, and

$$
K \geq\left[M^{3}+\frac{13 M N}{12 \beta}+\frac{5 L}{36 \beta^{2}}\right]^{1 / 3} .
$$

Let $h=K \beta \eta$. If $h \leq \frac{1}{2}$, then polynomial equation $g(t)$ has two positive real roots

$$
t^{*}=\frac{1-\sqrt{1-2 h}}{h} \eta, t^{* *}=\frac{1+\sqrt{1-2 h}}{h} \eta .
$$

Now we consider the majorizing sequences $\left\{t_{n}\right\},\left\{s_{n}\right\}(n \geq 0)$,

$$
\left\{\begin{array}{c}
s_{n}=t_{n}-\frac{g\left(t_{n}\right)}{g^{\prime}\left(t_{n}\right)}, t_{0}=0 \\
h_{n}=-g^{\prime}\left(t_{n}\right)^{-1} g^{\prime \prime}\left(r_{n}\right)\left(s_{n}-t_{n}\right) \\
t_{n+1}=t_{n}-\left[1+\frac{1}{2} h_{n}+\frac{1}{2} h_{n}^{2}\right] \frac{g\left(t_{n}\right)}{g^{\prime}\left(t_{n}\right)}
\end{array}\right.
$$

where $r_{n}=t_{n}+\frac{1}{3}\left(s_{n}-t_{n}\right)$.

Lemma 2. Suppose the sequences $t_{n}, s_{n}$ are generated by (13), if $h<\frac{1}{2}-$ $\frac{1}{2}\left(\frac{\sqrt[3]{5}-1}{\sqrt[3]{5}+1}\right)^{2}$, then the sequences $t_{n}$ and $s_{n}$ increase and converge to $t^{*}$, and

$$
0 \leq t_{n} \leq s_{n} \leq t_{n+1}<t^{*}
$$

Furthermore, we have

$$
t^{*}-t_{n} \leq \frac{\left(1-\theta^{2}\right) \eta}{1-\frac{1}{\sqrt[3]{5}}[\sqrt[3]{5} \theta]^{4^{n}}}[\sqrt[3]{5} \theta]^{4^{n}-1}, n=0,1, \cdots
$$


where $\theta=t^{*} / t^{* *}=(1-\sqrt{1-2 h}) /(1+\sqrt{1-2 h})$.

Proof. Let $a_{n}=t^{*}-t_{n}, b_{n}=t^{* *}-t_{n}$, we have

$$
\begin{aligned}
g\left(t_{n}\right) & =\frac{K}{2}\left(t^{*}-t_{n}\right)\left(t^{* *}-t_{n}\right)=\frac{K}{2} a_{n} b_{n}, \\
g^{\prime}\left(t_{n}\right) & =-\frac{K}{2}\left[\left(t^{*}-t_{n}\right)+\left(t^{* *}-t_{n}\right)\right]=-\frac{K}{2}\left(a_{n}+b_{n}\right) .
\end{aligned}
$$

We can obtain

$$
\begin{gathered}
s_{n}-t_{n}=-\frac{g\left(t_{n}\right)}{g^{\prime}\left(t_{n}\right)}=\frac{a_{n} b_{n}}{a_{n}+b_{n}}, \\
t_{n+1}-t_{n}=-\left[1+\frac{1}{2} h_{n}+\frac{1}{2} h_{n}^{2}\right] \frac{g\left(t_{n}\right)}{g^{\prime}\left(t_{n}\right)} \\
=\frac{\left(a_{n}+b_{n}\right)^{4}+a_{n} b_{n}\left(a_{n}+b_{n}\right)^{2}+2 a_{n}^{2} b_{n}^{2}}{\left(a_{n}+b_{n}\right)^{4}} \cdot \frac{a_{n} b_{n}}{a_{n}+b_{n}},
\end{gathered}
$$

and

$$
\begin{aligned}
t_{n+1}-s_{n} & =t_{n+1}-t_{n}-\left(s_{n}-t_{n}\right) \\
& =\frac{a_{n} b_{n}\left(a_{n}+b_{n}\right)^{2}+2 a_{n}^{2} b_{n}^{2}}{\left(a_{n}+b_{n}\right)^{4}} \cdot \frac{a_{n} b_{n}}{a_{n}+b_{n}} .
\end{aligned}
$$

Therefore, we have

$$
\begin{aligned}
a_{n+1} & =t^{*}-t_{n+1}=t^{*}-t_{n}-\left(t_{n+1}-t_{n}\right) \\
& =\frac{a_{n}^{4}\left(a_{n}^{2}+4 a_{n} b_{n}+5 b_{n}^{2}\right)}{\left(a_{n}+b_{n}\right)^{5}},
\end{aligned}
$$

and

$$
\begin{aligned}
b_{n+1} & =t^{* *}-t_{n+1}=t^{* *}-t_{n}-\left(t_{n+1}-t_{n}\right) \\
& =\frac{b_{n}^{4}\left(b_{n}^{2}+4 a_{n} b_{n}+5 a_{n}^{2}\right)}{\left(a_{n}+b_{n}\right)^{5}} .
\end{aligned}
$$

By (16)-(20), $t_{0}=0<t^{*}$, and by induction, we know (14) holds.

From (19) and (20), we get

$$
\frac{t^{*}-t_{n+1}}{t^{* *}-t_{n+1}} \leq 5\left[\frac{t^{*}-t_{n}}{t^{* *}-t_{n}}\right]^{4} .
$$

Because $t^{* *}-t_{n}=t^{*}-t_{n}+t^{* *}-t^{*}=t^{*}-t_{n}+\left(1-\theta^{2}\right) \eta / \theta$, we easily see that 


$$
t^{*}-t_{n} \leq \frac{\left(1-\theta^{2}\right) \eta}{1-\frac{1}{\sqrt[3]{5}}[\sqrt[3]{5} \theta]^{4^{n}}}[\sqrt[3]{5} \theta]^{4^{n}-1}, n=0,1, \cdots
$$

And since $\sqrt[3]{5} \theta<1$, we get $t_{n}$ and $s_{n}$ increase and converge to $t^{*}$. That completes the proof of the lemma 2 .

Lemma 3. Under the above assumptions and the sequences $t_{n}, s_{n}$ are generated by (13). Then the following items are true for all $n \geq 0$ :

(I) $\left\|x_{n}-x_{0}\right\| \leq t_{n}$

(II) $\left\|F^{\prime}\left(x_{n}\right)^{-1}\right\| \leq-g^{\prime}\left(t_{n}\right)^{-1}$,

$(I I I)\left\|y_{n}-x_{n}\right\| \leq s_{n}-t_{n}$,

$(I V)\left\|x_{n+1}-y_{n}\right\| \leq t_{n+1}-s_{n}$,

(V) $\left\|x_{n+1}-x_{n}\right\| \leq t_{n+1}-t_{n}$.

Proof. Estimate (I)-(V) are true for $n=0$ by initial conditions. Now assume that (I)-(V) are true for all integer $k \leq n$.

(I) From the above assumptions, we have

$$
\left\|x_{n+1}-x_{0}\right\| \leq\left\|x_{n+1}-x_{n}\right\|+\left\|x_{n}-x_{0}\right\| \leq t_{n+1}-t_{n}+t_{n}=t_{n+1} .
$$

(II) By condition (C3) and (12), we can obtain

$$
\begin{aligned}
\left\|F^{\prime}\left(x_{n+1}\right)-F^{\prime}\left(x_{0}\right)\right\| & \leq M\left\|x_{n+1}-x_{0}\right\| \leq M t_{n+1}<K t^{*} \\
& =K \eta \frac{1-\sqrt{1-2 h}}{h} \leq \frac{1}{\beta} \leq \frac{1}{\left\|F^{\prime}\left(x_{0}\right)^{-1}\right\|} .
\end{aligned}
$$

By Neumann Lemma, we get $F^{\prime}\left(x_{n+1}\right)^{-1}$ exists, and

$$
\begin{aligned}
& \leq \frac{\left\|F^{\prime}\left(x_{n+1}\right)^{-1}\right\|}{1-\left\|F^{\prime}\left(x_{0}\right)^{-1}\right\|\left\|F^{\prime}\left(x_{n+1}\right)-F^{\prime}\left(x_{0}\right)\right\|} \leq \frac{\beta}{1-\beta M\left\|x_{n+1}-x_{0}\right\|} \\
& \leq \frac{\beta}{1-\beta K t_{n+1}} \leq \frac{1}{\frac{1}{\beta}-K t_{n+1}}=-g^{\prime}\left(t_{n+1}\right)^{-1} .
\end{aligned}
$$

(III) By lemma 1 and using induction hypothesis, one has 


$$
\begin{aligned}
& F\left(x_{n+1}\right) \\
& \leq \frac{N}{6}\left\|y_{n}-x_{n}\right\|^{3}\left\|K_{F}\left(x_{n}\right)\right\|+\frac{M}{2}\left\|y_{n}-x_{n}\right\|^{2}\left\|K_{F}\left(x_{n}\right)\right\|^{2} \\
& +\frac{M}{2}\left\|x_{n+1}-y_{n}\right\|^{2}+\frac{5 L}{72}\left\|y_{n}-x_{n}\right\|^{4}+\frac{N}{2}\left\|y_{n}-x_{n}\right\|^{2}\left\|x_{n+1}-y_{n}\right\| \\
& \leq \frac{M}{2}\left[\left\|K_{F}\left(x_{n}\right)\right\|^{2}\left\|y_{n}-x_{n}\right\|^{2}+\left\|x_{n+1}-y_{n}\right\|^{2}\right]+\frac{N}{6}\left\|y_{n}-x_{n}\right\|^{3}\left\|K_{F}\left(x_{n}\right)\right\| \\
& +\frac{5 L}{72}\left\|y_{n}-x_{n}\right\|^{4}+\frac{N}{2}\left\|y_{n}-x_{n}\right\|^{2}\left\|x_{n+1}-y_{n}\right\| \\
& \leq \frac{M}{2}\left\{\left\|x_{n+1}-y_{n}\right\|^{2}+\frac{M^{2}\left\|y_{n}-x_{n}\right\|^{4}}{\left[\frac{1}{\beta}-M\left\|x_{n}-x_{0}\right\|\right]^{2}}\right\}+\frac{N M}{6} \frac{\left\|y_{n}-x_{n}\right\|^{4}}{\frac{1}{\beta}-M\left\|x_{n}-x_{0}\right\|} \\
& +\frac{5 L}{72}\left\|y_{n}-x_{n}\right\|^{4}+\frac{N}{2}\left\|y_{n}-x_{n}\right\|^{2}\left\|x_{n+1}-y_{n}\right\| \\
& \leq \frac{M}{2}\left\|x_{n+1}-y_{n}\right\|^{2}+\left[M^{3}+\frac{13 M N}{12 \beta}+\frac{5 L}{36 \beta^{2}}\right] \cdot \frac{1}{2} \cdot \frac{\left\|y_{n}-x_{n}\right\|^{4}}{\left\{\frac{1}{\beta}-M\left\|x_{n}-x_{0}\right\|\right\}^{2}} \\
& \leq \frac{K}{2}\left(t_{n+1}-s_{n}\right)^{2}+\frac{1}{2} \cdot \frac{K^{3}\left(s_{n}-t_{n}\right)^{4}}{\left\{\frac{1}{\beta}-K t_{n}\right\}^{2}}=g\left(t_{n+1}\right) .
\end{aligned}
$$

Hence, we get

$$
\begin{aligned}
\left\|y_{n+1}-x_{n+1}\right\| & =\left\|-F^{\prime}\left(x_{n+1}\right)^{-1} F\left(x_{n+1}\right)\right\| \\
& \leq-\frac{g\left(t_{n+1}\right)}{g^{\prime}\left(t_{n+1}\right)}=s_{n+1}-t_{n+1} .
\end{aligned}
$$

(IV) Since

$$
\begin{aligned}
\left\|I-K_{F}\left(x_{n+1}\right)\right\| & \geq 1-\left\|K_{F}\left(x_{n+1}\right)\right\| \geq 1-\frac{1}{2}\left(-g^{\prime}\left(t_{n+1}\right)\right)^{-1} g^{\prime \prime}\left(r_{n+1}\right)\left(s_{n+1}-t_{n+1}\right) \\
& =1+\frac{1}{2} g^{\prime}\left(t_{n+1}\right)^{-1} g^{\prime \prime}\left(r_{n+1}\right)\left(s_{n+1}-t_{n+1}\right)=1-h_{n+1},
\end{aligned}
$$

then

$$
\begin{aligned}
& \left\|x_{n+2}-y_{n+1}\right\| \\
= & \frac{1}{2}\left\|K_{F}\left(x_{n+1}\right)\left[I-K_{F}\left(x_{n+1}\right)\right]^{-1}\right\|\left\|F^{\prime}\left(x_{n+1}\right)^{-1} F\left(x_{n+1}\right)\right\| \\
\leq & \frac{1}{2} \frac{h_{n+1}}{1-h_{n+1}} \cdot \frac{g\left(t_{n+1}\right)}{-g^{\prime}\left(t_{n+1}\right)}=t_{n+2}-s_{n+1} .
\end{aligned}
$$

(V) From (24) and (25), we can obtain

$$
\begin{aligned}
\left\|x_{n+2}-x_{n+1}\right\| & \leq\left\|x_{n+2}-y_{n+1}\right\|+\left\|y_{n+1}-x_{n+1}\right\| \\
& \leq t_{n+2}-t_{n+1} .
\end{aligned}
$$


Theorem 1. Let $X$ and $Y$ be two Banach spaces and $F: \Omega \subseteq X \rightarrow Y$ be a third order Fréchet differentiable on a non-empty open convex subset $\Omega$. Assume that all conditions (C1)-(C5) hold and $x_{0} \in \Omega$. If $h=K \beta \eta<\frac{1}{2}-\frac{1}{2}\left(\frac{\sqrt[3]{5}-1}{\sqrt[3]{5}+1}\right)^{2}, \overline{B\left(x_{0}, t^{*}\right)} \in$ $\Omega$, then the sequence $\left\{x_{n}\right\}$ generated by (5) is well defined, $\left\{x_{n}\right\} \in \overline{B\left(x_{0}, t^{*}\right)}$ and converges to the unique solution $x^{*} \in B\left(x_{0}, t^{* *}\right)$ of $F(x)$, and $\left\|x_{n}-x^{*}\right\| \leq t^{*}-t_{n}$. Furthermore, we have

$$
\left\|x^{*}-x_{n}\right\| \leq \frac{\left(1-\theta^{2}\right) \eta}{1-\frac{1}{\sqrt[3]{5}}[\sqrt[3]{5} \theta]^{4^{n}}}[\sqrt[3]{5} \theta]^{4^{n}-1}, n=0,1, \cdots
$$

where $\theta=t^{*} / t^{* *}=(1-\sqrt{1-2 h}) /(1+\sqrt{1-2 h})$.

Proof. From the lemma 3, we can obtain that the sequence $\left\{x_{n}\right\}_{n}>0$ generated by (5) is well defined, $x_{n} \in \overline{B\left(x_{0}, t^{*}\right)}$ and converges to the solution $x^{*} \in \overline{B\left(x_{0}, t^{*}\right)}$ of $F(x)$.

Now we prove the uniqueness. Suppose $y^{*}$ also is the solution of $F(x)$ on $B\left(x_{0}, t^{* *}\right)$. Then, we have

$$
\begin{aligned}
& \left\|F^{\prime}\left(x_{0}\right)^{-1} \int_{0}^{1} F^{\prime}\left(x^{*}+t\left(y^{*}-x^{*}\right)\right) d t-I\right\| \\
\leq & \left\|F^{\prime}\left(x_{0}\right)^{-1}\right\|\left\|\int_{0}^{1}\left[F^{\prime}\left(x^{*}+t\left(y^{*}-x^{*}\right)\right)-F^{\prime}\left(x_{0}\right)\right] d t\right\| \\
\leq & M \beta \int_{0}^{1}\left\|x^{*}+t\left(y^{*}-x^{*}\right)-x_{0}\right\| d t \\
\leq & M \beta \int_{0}^{1}\left[(1-t)\left\|x^{*}-x_{0}\right\|+t\left\|y^{*}-x_{0}\right\|\right] d t \\
< & \frac{M \beta}{2}\left(t^{*}+t^{* *}\right) \leq 1 .
\end{aligned}
$$

By Neumann Lemma, we get the inverse of $\int_{0}^{1} F^{\prime}\left[x^{*}+t\left(y^{*}-x^{*}\right)\right] d t$ exists. Because

$$
0=F\left(y^{*}\right)-F\left(x^{*}\right)=\int_{0}^{1} F^{\prime}\left(x^{*}+t\left(y^{*}-x^{*}\right)\right) d t\left(y^{*}-x^{*}\right),
$$

so, $y^{*}=x^{*}$. That completes the proof of the unique solution. Moreover, when $m>n$, (28) $\left\|x_{m}-x_{n}\right\| \leq\left\|x_{m}-x_{m-1}\right\|+\left\|x_{m-1}-x_{m-2}\right\|+\cdots+\left\|x_{n+1}-x_{n}\right\| \leq t_{m}-t_{n}$, and let $m \rightarrow \infty$, we get

$$
\left\|x_{n}-x^{*}\right\| \leq t^{*}-t_{n} .
$$

From the lemma 2, we obtain 


$$
\left\|x^{*}-x_{n}\right\| \leq \frac{\left(1-\theta^{2}\right) \eta}{1-\frac{1}{\sqrt[3]{5}}[\sqrt[3]{5} \theta]^{4^{n}}}[\sqrt[3]{5} \theta]^{4^{n}-1}, n=0,1, \cdots .
$$

This completes the proof of the theorem.

\section{Local Convergence}

Assume that $F: D \subset X \rightarrow Y$ has the following property: there is an $x^{*} \in D$ such that

$$
F\left(x^{*}\right)=0,
$$

and $F^{\prime}\left(x^{*}\right)$ is nonsingular.

Theorem 2. Assume that the operate $F$ satisfies (C3)-(C5) and there exist $\delta$ and $\beta^{*}$ such that

$$
\left\|x_{n}-x^{*}\right\| \leq \delta \text { and }\left\|F^{\prime}\left(x^{*}\right)^{-1}\right\| \leq \beta^{*}
$$

then $x_{n+1}$ is well defined and converges to $x^{*}$ with order of convergence four. We also have the following error estimate:

$$
\left\|x_{n+1}-x^{*}\right\| \leq C^{*}\left\|x_{n}-x^{*}\right\|^{4},
$$

where

$$
C^{*}=\frac{\left[\frac{M^{3} e^{* 2}}{2}+\frac{M^{3}}{2}+\frac{13 M N}{24 \beta^{*}}+\frac{5 L}{72 \beta^{* 2}}\right]}{\left[\frac{1}{\beta^{*}}-2 M \delta\right]^{2}} \frac{1+M \beta^{*} \delta}{\alpha},
$$

and $e^{*}=1+2 M \beta^{*} \delta+2\left(M \beta^{*} \delta\right)^{2}, \alpha>0$.

Proof. Since

$$
\left\|F^{\prime}\left(x_{n}\right)-F^{\prime}\left(x^{*}\right)\right\| \leq M\left\|x_{n}-x^{*}\right\| \leq M \delta,
$$

we can choose $\delta>0$ such that $\delta \leq \frac{1}{2 M \beta^{*}}$. Thus $F^{\prime}\left(x_{n}\right)^{-1}$ exists and

$$
\begin{aligned}
\left\|F^{\prime}\left(x_{n}\right)^{-1}\right\| & \leq \frac{\left\|F^{\prime}\left(x^{*}\right)^{-1}\right\|}{1-\left\|F^{\prime}\left(x^{*}\right)^{-1}\right\|\left\|F^{\prime}\left(x_{n}\right)-F^{\prime}\left(x^{*}\right)\right\|} \\
& \leq \frac{\beta^{*}}{1-\beta^{*} M \delta}=\frac{1}{\frac{1}{\beta^{*}}-M \delta} \leq 2 \beta^{*},
\end{aligned}
$$

then

$$
\left\|K_{F}\left(x_{n}\right)\right\| \leq\left\|F^{\prime}\left(x_{n}\right)^{-1}\right\|\left\|F^{\prime \prime}\left(u_{n}\right)\right\|\left\|y_{n}-x_{n}\right\| \leq 2 M \beta^{*}\left\|y_{n}-x_{n}\right\| .
$$

We also have 


$$
\begin{aligned}
\left\|x_{n+1}-y_{n}\right\| & \leq \frac{1}{2}\left\|K_{F}\left(x_{n}\right)\right\|\left[1+\left\|K_{F}\left(x_{n}\right)\right\|\right]\left\|y_{n}-x_{n}\right\| \\
& \leq \frac{M \beta^{*}\left\|y_{n}-x_{n}\right\|^{2}}{1-2 M \beta^{*}\left\|x_{n}-x^{*}\right\|}\left[1+2 M \beta^{*}\left\|y_{n}-x_{n}\right\|\right] \\
& \leq \frac{M\left\|y_{n}-x_{n}\right\|^{2}}{\frac{1}{\beta^{*}}-2 M\left\|x_{n}-x^{*}\right\|}\left[1+2 M \beta^{*} \delta+2\left(M \beta^{*} \delta\right)^{2}\right] .
\end{aligned}
$$

From the lemma 1, we have

$$
\begin{aligned}
& \left\|F\left(x_{n+1}\right)\right\| \\
\leq & \frac{M}{2}\left\|x_{n+1}-y_{n}\right\|^{2}+\left[M^{3}+\frac{13 M N}{12 \beta^{*}}+\frac{5 L}{36 \beta^{* 2}}\right] \cdot \frac{1}{2} \cdot \frac{\left\|y_{n}-x_{n}\right\|^{4}}{\left[\frac{1}{\beta^{*}}-M\left\|x_{n}-x^{*}\right\|\right]^{2}} \\
\leq & \frac{M^{3} e^{* 2}}{2} \frac{\left\|y_{n}-x_{n}\right\|^{4}}{\left[\frac{1}{\beta^{*}}-2 M\left\|x_{n}-x^{*}\right\|\right]^{2}}+\frac{\left[\frac{13 M N}{2}+\frac{5 L}{72 \beta^{* 2}}\right]\left\|y_{n}-x_{n}\right\|^{4}}{\left[\frac{1}{\beta^{*}}-M\left\|x_{n}-x^{*}\right\|\right]^{2}} \\
\leq & \frac{\left[\frac{M^{3} e^{* 2}}{2}+\frac{M^{3}}{2}+\frac{13 M N}{24 \beta^{*}}+\frac{5 L}{72 \beta^{* 2}}\right]}{\left[\frac{1}{\beta^{*}}-2 M \delta\right]^{2}}\left\|y_{n}-x_{n}\right\|^{4} \\
= & C\left\|y_{n}-x_{n}\right\|^{4}
\end{aligned}
$$

where

$$
C=\frac{\frac{M^{3} e^{* 2}}{2}+\frac{M^{3}}{2}+\frac{13 M N}{24 \beta^{*}}+\frac{5 L}{72 \beta^{* 2}}}{\left[\frac{1}{\beta^{*}}-2 M \delta\right]^{2}} .
$$

On the other hand, by the continuity of $F^{\prime}\left(x^{*}\right)^{-1}$, there is an $\alpha$ such that

$$
\left\|F\left(x_{n+1}\right)-F\left(x^{*}\right)\right\| \geq \alpha\left\|x_{n+1}-x^{*}\right\|,
$$

and so

$$
\begin{aligned}
\frac{\left\|F\left(x_{n+1}\right)\right\|}{\left\|y_{n}-x_{n}\right\|^{4}} & =\frac{\left\|F\left(x_{n+1}\right)-F\left(x^{*}\right)\right\|}{\left\|y_{n}-x_{n}\right\|^{4}} \geq \frac{\alpha\left\|x_{n+1}-x^{*}\right\|}{\left\|y_{n}-x_{n}\right\|^{4}} \\
& \geq \frac{\alpha\left\|x_{n+1}-x^{*}\right\|}{\left[\left\|y_{n}-x^{*}\right\|+\left\|x_{n}-x^{*}\right\|\right]^{4}}=\alpha \frac{\frac{\left\|x_{n+1}-x^{*}\right\|}{\left\|x_{n}-x^{*}\right\|^{4}}}{\left[1+\frac{\left\|y_{n}-x^{*}\right\|}{\left\|x_{n}-x^{*}\right\|}\right]^{4}} .
\end{aligned}
$$

It follows that

$$
\begin{aligned}
\frac{\left\|x_{n+1}-x^{*}\right\|}{\left\|x_{n}-x^{*}\right\|^{4}} & \leq \frac{1}{\alpha}\left[1+\frac{\left\|y_{n}-x^{*}\right\|}{\left\|x_{n}-x^{*}\right\|}\right]^{4} \frac{\left\|F\left(x_{n+1}\right)\right\|}{\left\|y_{n}-x_{n}\right\|^{4}} \\
& =C \cdot \frac{1}{\alpha} \cdot\left[1+\frac{\left\|y_{n}-x^{*}\right\|}{\left\|x_{n}-x^{*}\right\|}\right]^{4}
\end{aligned}
$$


In view of the identity

$$
x^{*}-x_{n}=-F^{\prime}\left(x_{n}\right)^{-1} \int_{0}^{1} F^{\prime \prime}\left[x^{*}+t\left(x_{n}-x^{*}\right)\right] t d t\left(x^{*}-x_{n}\right)^{2},
$$

we get

$$
\left\|y_{n}-x^{*}\right\| \leq M \beta^{*}\left\|x_{n}-x^{*}\right\|^{2} .
$$

Hence, we deduce

$$
\begin{aligned}
\frac{\left\|x_{n+1}-x^{*}\right\|}{\left\|x_{n}-x^{*}\right\|} & \leq \frac{C}{\alpha}\left[1+M \beta^{*}\left\|x_{n}-x^{*}\right\|\right] \\
& \leq \frac{C}{\alpha}\left(1+M \beta^{*} \delta\right)=C^{*} .
\end{aligned}
$$

This completes the proof of the theorem.

\section{Applications}

In this section, we illustrate the previous study with an application to the following nonlinear integral equations.

We now consider the nonlinear integral equation $F(x)=0$, where

$$
F(x)(s)=x(s)-4 / 3+\frac{1}{2} \int_{0}^{1} s \cos (x(t)) d t,
$$

where $s \in[0,1]$ and $x \in \Omega=B(0,2) \subset X$. Here, $X=C[0,1]$ is the space of continuous functions on $[0,1]$ with the max-norm,

$$
\|x\|=\max _{s \in[0,1]}|x(s)|
$$

We can obtain the derivatives of $F$ given by

$$
\begin{gathered}
F^{\prime}(x) y(s)=y(s)-\frac{1}{2} \int_{0}^{1} s \sin (x(t)) y(t) d t, y \in \Omega, \\
F^{\prime \prime}(x) y z(s)=-\frac{1}{2} \int_{0}^{1} s \cos (x(t)) y(t) z(t) d t, y, z \in \Omega, \\
F^{\prime \prime \prime}(x) y z u(s)=\frac{1}{2} \int_{0}^{1} s \sin (x(t)) y(t) z(t) u(t) d t, y, z, u \in \Omega .
\end{gathered}
$$

Furthermore, we have

$$
\begin{gathered}
\left\|F^{\prime \prime}(x)\right\| \leq \frac{1}{2} \equiv M, x \in \Omega, \\
\left\|F^{\prime \prime \prime}(x)\right\| \leq \frac{1}{2} \equiv N, x \in \Omega,
\end{gathered}
$$


and the Lipschitz condition with $L=\frac{1}{2}$

$$
\left\|F^{\prime \prime \prime}(x)-F^{\prime \prime \prime}(y)\right\| \leq \frac{1}{2}\|x-y\|, x, y \in \Omega .
$$

A constant function, i.e. $x_{0}(t)=4 / 3$, is chosen as the initial approximate solution. It follows that

$$
\left\|F\left(x_{0}\right)\right\| \leq \frac{1}{2} \cos (4 / 3) \text {. }
$$

In this case, we have

$$
\left\|I-F^{\prime}\left(x_{0}\right)\right\| \leq \frac{1}{2} \sin (4 / 3),
$$

and then by the Banach lemma, we include that $\Gamma_{0}$ exists and obtain

$$
\left\|\Gamma_{0}\right\| \leq \frac{2}{2-\sin (4 / 3)} \equiv \beta,\left\|\Gamma_{0} F\left(x_{0}\right)\right\| \leq \frac{\cos (4 / 3)}{2-\sin (4 / 3)} \equiv \eta \text { and } K=0.6562 .
$$

Note that $h=K \beta \eta=0.2921<\frac{1}{2}-\frac{1}{2}\left(\frac{\sqrt[3]{5}-1}{\sqrt[3]{5}+1}\right)^{2}$, therefore $t^{*}=0.2782, t^{* *}=$ 1.2884. This means that the hypotheses of Theorem 1 is satisfied. Hence the solution $x^{*}$ belongs to $\overline{B\left(x_{0}, R \eta\right)}=\overline{B(4 / 3,0.2782)} \subseteq \Omega$ and it is unique in $B(4 / 3,1.2884) \cap \Omega$.

Using the trapezoidal rule of integration with step $h=1 / m$ to discretize (36), we obtain the following system of nonlinear equations

$$
0=x_{i}-4 / 3+\frac{s_{i}}{2 m}\left(\frac{1}{2} \cos \left(x_{0}\right)+\sum_{j=1}^{m-1} \cos \left(x_{j}\right)+\frac{1}{2} \cos \left(x_{m}\right)\right), i=0,1, \cdots, m,
$$

where $s_{i}=t_{i}=i / m$ and $x_{i}=x\left(t_{i}\right)$.

Now we apply the modified Chebyshev-like's method given by (5) to compute (37) and compare it with the Chebyshev-like's method. We give the initial guess $x_{i}=4 / 3, i=0,1, \ldots, m$. All computations are carried out with double arithmetic precision. In the tests, we take $m=10,15,20$ in (37) respectively. Displayed in Tables 2, 3 and 4 is the max-norm of vector functions at each iterative step. The stopping criterion that we consider is $\left\|F\left(x_{m}\right)\right\|_{\infty} \leq 10^{-15}$.

On the other hand, we analyze computational cost for these methods with Chebyshevlike's method in [28], super-Halley's method in [12] and the modified Chebyshev-like's method (5), when they are applied to solve (37). Table 5 shows the comparison results for computational cost. We observe that the cost of the super-Halley's method in [12] is expensive. Compared with the Chebyshev-like's method in [28], the method (5) requires nearly the same costs, but the convergence speed of method (5) is fast.

From the numerical results, we can see that the performance of our method is better. This means that our method can be of practical interest. 
Table 2: Results of system (37) with $m=10$

\begin{tabular}{lcc}
\hline $\mathrm{n}$ & Chebyshev-like's method & Modified Chebyshev-like's method \\
\hline 1 & $7.519390 \mathrm{e}-005$ & $5.170680 \mathrm{e}-007$ \\
2 & $1.860149 \mathrm{e}-014$ & $1.266747 \mathrm{e}-016$ \\
3 & $1.040409 \mathrm{e}-016$ & \\
\hline
\end{tabular}

Table 3: Results of system (37) with $m=15$

\begin{tabular}{lcc}
\hline $\mathrm{n}$ & Chebyshev-like's method & Modified Chebyshev-like's method \\
\hline 1 & $7.478519 \mathrm{e}-005$ & $5.124295 \mathrm{e}-007$ \\
2 & $1.838788 \mathrm{e}-014$ & $1.247163 \mathrm{e}-016$ \\
3 & $1.077939 \mathrm{e}-016$ & \\
\hline
\end{tabular}

Table 4: Results of system (37) with $m=20$

\begin{tabular}{lcc}
\hline $\mathrm{n}$ & Chebyshev-like's method & Modified Chebyshev-like's method \\
\hline 1 & $7.464214 \mathrm{e}-005$ & $5.108072 \mathrm{e}-007$ \\
2 & $1.830851 \mathrm{e}-014$ & $1.901605 \mathrm{e}-016$ \\
3 & $1.065761 \mathrm{e}-016$ & \\
\hline
\end{tabular}

Table 5: Computational cost per iteration when these methods are applied to solve a nonlinear system of $m$ equations

\begin{tabular}{cccc}
\hline Method & $m$ & Number of computations of functions & LR-dec. \\
\hline \multirow{3}{*}{ Chebyshev-like's method } & 10 & 660 & 1 \\
& 15 & 2040 & 1 \\
& 20 & 4620 & 1 \\
\hline \multirow{3}{*}{ Super-Halley's method } & 10 & 660 & 2 \\
& 15 & 2040 & 2 \\
& 20 & 4620 & 2 \\
\hline \multirow{3}{*}{ Method (5) } & 10 & 660 & 1 \\
& 15 & 2040 & 1 \\
\hline
\end{tabular}




\section{CONCLUSION}

In this paper, a modified Chebyshev-like's method with fourth-order is introduced and the semilocal convergence of the method for solving nonlinear equations in Banach spaces is established by using majorizing functions. An existence-uniqueness theorem is given to show the R-order convergence of the method. Moreover, the local convergence of the method is also analyzed. From the numerical results, we can observe that the performance of our method is better.

\section{REFERENCES}

1. J. M. Ortega and W. C. Rheinbolt, Iterative Solution of Nonlinear Equations in Several Variables, Academic Press, New York, 1970.

2. V. Candela and A. Marquina, Recurrence relations for rational cubic methods I: the Halley method, Computing, 44 (1990), 169-184.

3. V. Candela and A. Marquina, Recurrence relations for rational cubic methods II: the Chebyshev method, Computing, 45 (1990), 355-367.

4. J. M. Gutiérrez and M. A. Hernández, Recurrence relations for the super-Halley method, Comput. Math. Appl., 36 (1998), 1-8.

5. P. K. Parida and D. K. Gupta, Semilocal convergence of a family of a third-order Chebyshev-type methods under a mild differentiability condition, Int. J. Comput. Math., 87 (2010), 3405-3419.

6. P. K. Parida and D. K. Gupta, Recurrence relations for a Newton-like method in Banach spaces, J. Comput. Appl. Math., 206 (2007), 873-887.

7. S. Amat, S. Busquier and J. M. Gutierrez, Third-order iterative methods with applications to Hammerstein equations: a unified approach, J. Comput. Appl. Math., 235 (2011), 2936-2943.

8. M. A. Hernández and N. Romero, General study of iterative processes of R-order at least three under general convergence conditions, J. Optim. Theory Appl., 133 (2007), 163-177.

9. S. Amat, C. Bermúdez, S. Busquier and S. Plaza, On a third-order Newton-type method free of bilinear operators, Numer. Linear Algebra Appl., 17 (2010), 639-653.

10. J. A. Ezquerro and M. A. Hernández, On the R-order of the Halley method, J. Math. Anal. Appl., 303 (2005), 591-601.

11. J. A. Ezquerro and M. A. Hernández, Fourth-order iterations for solving Hammerstein integral equations, Appl. Numer. Math., 59 (2009), 1149-1158.

12. X. H. Wang, C. Q. Gu and J. S. Kou, Semilocal convergence of a multipoint fourth-order super-Halley method in Banach spaces, Numer. Algor., 56 (2011), 497-516. 
13. J. A. Ezquerro, J. M. Gutiérrez, M. A. Hernández and M. A. Salanova, The application of an inverse-free Jarratt-type approximation to nonlinear integral equations of Hammerstein-type, Comput. Math. Appl., 36 (1998), 9-20.

14. J. A. Ezqurro and M. A. Hernández, New iterations of R-order four with reduced compuational cost, BIT Numer. Math., 49 (2009), 325-342.

15. X. H. Wang, J. S. Kou and C. Q. Gu, Semilocal convergence of a class of modified super-Hallay methods in Banach spaces, J. Optim. Theory Appl., 153 (2012), 779-793.

16. X. H. Wang, Convergence of Newton's method and uniqueness of the solution of equations in Banach spaces II , Acta Math. Sin. (English Series), 19 (2003), 405-412.

17. I. K. Argyros, On the Newton-Kantorovich hypothesis for solving equations, J. Comput. Appl. Math., 169 (2004), 315-332.

18. I. K. Argyros, On the comparison of a weak variant of the Newton-Kantorovich and Miranda theorems, J. Comput. Appl. Math., 166 (2004), 585-589.

19. J. M. Gutiérrez and M. A. Hernández, A family of Chebshev-Halley type methods in Banach spaces, Bull. Austral. Math. Soc., 55 (1997), 113-130.

20. I. K. Argyros, Y. J. Cho and S. Hilout, On the semilocal convergence of the Halley method using recurrent functions, J. Appl. Math. Comput., 37 (2011), 221-246.

21. Q. B. Wu and Y. Q. Zhao, The convergence theorem for a famliy deformed Chebyshev method in Banach space, Appl. Math. Comput., 182 (2006), 1369-1376.

22. Q. B. Wu and Y. Q. Zhao, Newton-Kantorovich type convergence theorem for a famliy of new deformed Chebyshev method, Appl. Math. Comput., 192 (2007), 405-412.

23. J. H. Chen, I. K. Argyros and R. P. Agarwal, Majorizing function and two-point Newtontype method, J. Comput. Appl. Math., 234 (2010), 1473-1484.

24. Q. B. Wu and Y. Q. Zhao, Third-order convergence theorem by using majorizing function for a modified Newton method in Banach space, Appl. Math. Comput., 175 (2006), $1515-1524$.

25. I. K. Argyros and S. Hilout, Majorizing sequences for iterative methods, J. Comput. Appl. Math., 236 (2012), 1947-1960.

26. I. K. Argyros, D. Chen and Q. Qian, An inverse-free Jarratt type approximation in a Banach space, Approx. Theory \& Its Appl., 12 (1996), 19-30.

27. L. Zheng and C. Q. Gu, Fourth-order convergence theorem by using majorizing functions for super-Halley method in Banach spaces, International Journal of Computer Mathematics, 90 (2013), 423-434.

28. J. A. Ezquerro, J. M. Gutiérrez, M. A. Hernández and M. A. Salanova, Chebyshev-like methods and quadratic equations, Rev. Anal. Numér. Théor. Approx., 28 (1999), 23-25.

29. S. Amat, S. Busquier and J. M. Gutiérrez, An adaptative version of a fourth-order iterative method for quadratic equations, J. Comput. Appl. Math., 191 (2006), 259-268. 
30. J. S. Kou, Y. T. Li and X. H. Wang, A variant of super-Halley method with accelerated fourth-order convergence, Appl. Math. Comput., 186 (2007), 535-539.

Lin Zheng*1,2, Ke Zhang ${ }^{2}$ and Liang Chen ${ }^{2,3}$

${ }^{1}$ School of Statistics and Applied Mathematics Anhui University of Finance \& Economics Bengbu 233030

P. R. China

E-mail: hbzhenglin@126.com

${ }^{2}$ Department of Mathematics

Shanghai University, Shanghai 200444

P. R. China

${ }^{3}$ School of Mathematics Science

Huaibei Normal University

Huaibei $235000 \quad$ P. R. China 